\title{
ArcheoSciences
}

Revue d'archéométrie

$34 \mid 2010$

Varia

\section{Le plomb et l'argent dans le Gévaudan médiéval}

Lead and silver in the medieval Gévaudan

\section{Marie-Christine Bailly-Maître}

\section{(2) OpenEdition}

Journals

Édition électronique

URL : https://journals.openedition.org/archeosciences/2716

DOI : 10.4000/archeosciences. 2716

ISSN : 2104-3728

\section{Éditeur}

Presses universitaires de Rennes

\section{Édition imprimée}

Date de publication : 10 avril 2010

Pagination : 137-148

ISBN : 978-2-7535-1407-2

ISSN : 1960-1360

\section{Référence électronique}

Marie-Christine Bailly-Maître, «Le plomb et l'argent dans le Gévaudan médiéval », ArcheoSciences [En ligne], 34 | 2010, mis en ligne le 11 avril 2013, consulté le 28 janvier 2022. URL : http:// journals.openedition.org/archeosciences/2716 ; DOI : https://doi.org/10.4000/archeosciences.2716 


\title{
Le plomb et l'argent dans le Gévaudan médiéval

\author{
Lead and silver in the medieval Gévaudan
}

\author{
Marie-Christine BAILLY-MAîTRE*
}

\begin{abstract}
Résumé : Le Moyen Âge correspond à un temps fort de l'activité minière et métallurgique.Les minerais argentifères sont particulièrement recherchés car ils sont liés à la frappe monétaire, droit régalien exercé par les puissants seigneurs. La recherche historique et archéologique menée depuis trois décennies a considérablement fait progresser les connaissances sur les questions politiques, économiques, sociales, juridiques entourant l'activité minière. La fouille et l'étude de grands districts miniers méditerranéens a également apporté des informations inédites sur les techniques extractives et minières. Avec le programme centré sur le Mont Lozère, ce sont des aspects nouveaux qui sont abordés: techniques de la métallurgie du plomb argentifere, impact environnemental de cette activité, recherche des minerais source à partir de la filiation isotopique. Les sites à scories du Mont Lozère offrent un potentiel unique pour conduire ces recherches et deux d'entre eux ont concentré les travaux concertés des chercheurs oeuvrant dans le cadre du Projet Collectif de Recherche.
\end{abstract}

\begin{abstract}
The Middle Ages was a key period for mining and metallurgical activity. Silver-bearing ores were especially sought-after for the minting of coins, the regal right of the powerful lords. The historical and archaeological research carried out in the last three decades has considerably advanced knowledge regarding the political, economic, social and legal issues surrounding the mining activity. Excavation and investigation of large Mediterranean mining districts have also provided previously unknown information on extraction techniques. With the programme focused on Mont Lozère, new aspects are being addressed: metallurgy of argentiferous lead, environmental impact of the activity and the search for source ores based on isotopic genealogy/relationships. The Mont Lozere slag sites offer unique potential for conducting this research and two of them are the focal point of the joint work of researchers in the framework of the Collective Research Project.
\end{abstract}

Mots clé : Gévaudan, plomb, argent, fours, métallurgie, Moyen Âge.

Key words: Gévaudan, lead, silver, furnaces, metallurgy, Middle Ages.

\section{INTRODUCTION}

\section{Rappel du contexte de cette recherche}

Le Moyen Âge correspond à un temps fort de l'activité minière et métallurgique. La période qui va de la fin du $\mathrm{x}^{\mathrm{e}}$ au milieu du XIV siècle connaît un accroissement démographique important, une évolution des techniques agraires, architecturales et de l'artisanat, un développement du phénomène urbain, bref un accroissement des besoins et des échanges. À ce dynamisme doit répondre un dynamisme égal de la production de métal. Le fer entre dans l'outillage, la construction, l'armement, etc. Les usages du plomb et du cuivre sont considérables. L'argent est l'objet de l'attention des puissants car étroitement lié au monnayage.

Le champs de la recherche est large et prend en compte l'ensemble des phénomènes liés à l'activité minière et métallurgique : les rapports de force politiques et économiques, les incidences sur le plan social (localisation de l'habitat, évolution du statut des acteurs au fil des siècles), les flux des

* Laboratoire d'archéologie Médiévale Méditerranéenne UMR 6572 CNRS - Université de Provence - MMSH, 5 rue du château de l'Horloge, BP 647, 13094 Aix-en-Provence cedex 02. 
compétences, les grandes phases de mutations techniques, le poids des données naturelles sur le déroulement de l'activité (géologie et gîtologie, hydrologie, réserves en combustibles), l'impact de l'activité sur le paysage et l'environnement (modification de la topographie naturelle, pollution, déforestation), etc.

La connaissance de cette activité hautement stratégique dépend du croisement de sources différentes. L'histoire des techniques a tout à attendre de l'archéologie et de l'archéométrie. Le Moyen Âge n'a laissé aucun traité théorique ou pratique sur le sujet. C'est au XVI ${ }^{\mathrm{e}}$ siècle que sont publiés les premiers traités et encore concernent-ils l'Europe du Centre et de l'Est.

Seule une lecture croisée et une mise en perspective des données fournies par les textes, l'iconographie, l'archéologie, l'archéométrie, l'expérimentation et l'ethnoarchéologie permet d'écrire cette histoire des techniques et de la comprendre dans son contexte historique (Bailly-Maître, Dillman, à paraitre). Cette approche, globale et intégrée, amène à une relecture à la fois des sources documentaires (archives, traités), mais aussi à reconsidérer l'ensemble de la société médiévale.

\section{Les sources de l'histoire}

La recherche documentaire, préalable aux opérations sur le terrain et en laboratoire, se fonde sur des sources iconographiques, cartographiques et textuelles.

L'iconographie médiévale est rare dans ce domaine. Les exemples les plus fameux sont les vitraux de la cathédrale de Fribourg (1350), les vignettes illustrant le Lapidario d'Alphonse X (Castille - XIII ${ }^{e}$ siècle) ou encore les scènes du graduel de Kutna Hora (Bohême - fin $\mathrm{Xv}^{\mathrm{e}}$ siècle), et encore ne concernent-ils que l'extraction du minerai, en aucun cas sa métallurgie.

Les sources textuelles informent sur l'évolution du mode d'appropriation, les fluctuations des prix des métaux, les conflits pour la possession des gisements, le mode de gestion des entreprises, etc. (Braunstein, 1990; Hesse, 1968; Bailly-Maître, Gaucher, 2002; Bailly-Maître, Dhénin, 2004; Francovich, 1985; Bailly-Maître, Poisson, 2007).

À partir du terrain, l'historien/archéologue définit l'ampleur de l'activité et son impact environnemental; il contribue aussi à une histoire des techniques. Pour cela, il s'appuie sur la toponymie (Cros, Minière, Argentière et leurs dérivés), le contexte géologique et la topographie de surface. L'étude des réseaux miniers repose sur l'observation de la forme des chantiers, sur les traces d'abattage conservées en parois, sur la gestion de l'espace souterrain : exhaure, aération, circulation, éclairage. La transformation métallurgique des mine- rais laisse des signes forts sur le terrain. C'est aussi dans ce champs-là que l'apport de l'archéométrie est le plus essentiel (Fluzin et al., 2000).

\section{Les relations hommes-milieux}

L'activité extractive dépend de la présence de gisements, elle est donc prédéterminée géographiquement. Ce déterminisme est particulièrement prégnant pour les minerais argentifères généralement à l'origine d'infrastructures liées au fonctionnement de l'entreprise : aménagement du territoire, développement d'habitat, etc. La gîtologie conditionne la forme des chantiers - on n'exploite pas de la même façon un gisement stratiforme et filonien - de sorte que le milieu interfère avec l'activité humaine sur le plan technique.

La recherche sur l'activité minière et métallurgique conduit à une archéologie du paysage. Les exploitations laissent des traces indélébiles : modification des topographies de surface, des réseaux hydrographiques, création d'étangs, de chemins des mineurs, etc. Les haldes sont immédiatement perceptibles (absence de végétation, reliefs discordants par rapport à l'environnement) (Bailly-Maître, 2008).

L'impact environnemental se mesure également à la pollution générée par l'activité (Baron, 2005), au poids de l'activité sur le couvert forestier. Le bois est nécessaire aux équipements souterrains : planchers, étayages, échelles, cuvelage de puits; il est surtout charbonné pour les besoins de la métallurgie. Son exploitation intensive provoque des conflits d'intérêts, des réglementations dont les archives se font l'écho (Caliste, 2005).

\section{Le métal dans les sociétés médiévales : contribution à l'histoire}

Sur le plan de l'Histoire, il ne fait plus de doute, maintenant, que la possession de gisements miniers est un fait politique et stratégique, tout autant qu'économique et technique. Afin de s'assurer cette maîtrise, les puissants se sont affrontés, des spécialistes ont cherché à faire évoluer les techniques d'extraction et de transformation des minerais, et les progrès réalisés dans ces domaines ont eu des répercussions directes sur les autres secteurs d'activités : monnayage, armement, agriculture, artisanat, construction...

Le Moyen Âge «français » connaît, à partir du viI siècle, un quasi-monométallisme argent avec l'émission du denier et sa subdivision, l'obole, jusqu'au règne de Philippe IV le Bel. La conséquence est une recherche et une exploitation intensives des minerais argentifères.

La France méridionale se place alors dans les premiers rangs des producteurs de métal blanc. Les recherches ont 
mis en évidence de véritables politiques minières menées par de grandes familles pour le contrôle des districts argentiferes, politiques seigneuriales lentement battues en brèche par le grignotage capétien pour le contrôle des mines de métaux précieux qui se solde par l'ordonnance de 1413 (Isambert 1821-1833). Les acteurs de cette économie sont le comte de Toulouse, la vicomtesse de Narbonne, les familles de Trencavel, de Sauve et Anduze, le roi René, les évêques de Viviers, d'Embrun, de Mende, etc. Les bourgeois ne tardent guères à infiltrer cette activité tout à la fois périlleuse (les échecs sont nombreux) et très lucrative.

La naissance, au Moyen Âge, de bourgades ou de villes dont la disparition - Brandes à Huez - ou la survie jusqu'à nos jours - Largentière en Vivarais, l'Argentière en Embrunais, Saint-Laurent-le-Minier dans les Cévennes sont liées à la poursuite ou l'arrêt du fonctionnement des mines est la preuve manifeste de l'impact de cette activité sur l'occupation du sol et la structuration d'un territoire (Bailly-Maître, 2004).

Sur le plan de l'histoire des techniques, les apports sont inédits puisque produits par l'archéologie et l'archéométrie depuis une trentaine d'années.

\section{Le caractère exceptionnel du Mont-Lozère}

On sait peu de chose de la métallurgie du plomb ou du cuivre argentifères avant le $\mathrm{XVI}^{\mathrm{e}}$ siècle, moment où les traités techniques commencent à livrer des informations plus précises, mais qui restent encore pour une bonne part à interpréter (Agricola, 1556; Schwazerbergbuch, 1556; Biringuccio, 1540). La France est très en avance sur la recherche européenne, mais elle s'est surtout développée à partir de l'étude des sites extractifs des non ferreux et de la métallurgie du fer, faute de disposer d'un corpus valide pour les sites de métallurgie de plomb et de cuivre. C'est pourquoi, le Mont Lozère représente un potentiel inestimable.

Dans un premier temps, les prospections ont été menées à la recherche des "sites à scories " (Ploquin, article introductif dans ce volume). À ce jour, 86 sites ont été localisés et l'inventaire s'enrichit chaque année. Presque tous sont situés dans une aire d'environ $8 \mathrm{~km}^{2}$ et une bande altimétrique étroite (1360-1430 m). Les analyses pétrologiques ont montré qu'il s'agissait bien d'une métallurgie du plomb, très probablement de plomb argentifère (Mahé-Le Carlier, Ploquin, Fluck, dans ce volume). Les datations ${ }^{14} \mathrm{C}$ sur des charbons de bois associés aux scories ou en sole de charbonnières proches donnent une fourchette large qui va du début du XI ${ }^{\mathrm{e}}$ au début du XIV ${ }^{\mathrm{e}}$ siècle, mais pour l'essentiel elles sont groupées sur la période comprise entre le milieu du $\mathrm{XI}^{\mathrm{e}}$ siècle et la fin du XII ${ }^{\mathrm{e}}$ siècle. Du fait de la grande simili- tude des scories et de leurs gisements, nous sommes enclins à faire l'hypothèse que ces datations sont significatives pour la totalité du corpus. À ce jour, aucun ensemble comparable n'existe en France et plus généralement, en Europe.

Ce potentiel unique pour la connaissance de la métallurgie du plomb est enrichi par les possibilités d'études sur les minerais et l'environnement (voir Ploquin, Baron et al., dans ce volume).

Le grand nombre de sites médiévaux sur le Mont-Lozère, leur concentration dans un même écosystème plaident en faveur d'une activité limitée dans le temps, entre les $\mathrm{XI}^{\mathrm{e}}$ et XIII ${ }^{\mathrm{e}}$ siècles, moment de forte demande monétaire dans l'occident médiéval en plein essor économique. Le Gévaudan participe à ce mouvement général, malheureusement les documents le concernant font défaut avant les $\mathrm{XIII}^{\mathrm{e}}$ et $\mathrm{XIV}^{\mathrm{e}}$ siècles. La situation politique du Gévaudan est complexe. Durant la période qui nous concerne, $\mathrm{X}^{\mathrm{e}}-\mathrm{XIV}^{\mathrm{e}}$ siècles, la suzeraineté sur tout ou partie du Gévaudan a constamment changé entre les Maisons de Toulouse et d'Aragon ou Barcelone, mais est aussi disputée entre les barons, le Prieuré d'Ispagnac, l'évêque de Mende et enfin, le roi de France ${ }^{1}$.

Le Gévaudan médiéval comprenait huit baronnies, un comté, une vicomté, un diocèse. Dès le $\mathrm{xI}^{\mathrm{e}}$ siècle, le titre de comte échoit à des familles lointaines, qui s’en désintéressent, et les vicomtes profitent de cette situation. En face d'eux, l'évêque de Mende n'a de cesse que d'accroître son autorité sur le Gévaudan. L'évêque Aldeberg III du Tournel, issu de la grande famille des barons du Tournel, qui entre en fonction au milieu du $\mathrm{XII}^{\mathrm{e}}$ siècle, mène une politique de rachat de droits et de terres. En 1161, en échange de son allégeance, Louis VII lui octroie une charte, connue sous le nom de "Bulle d'Or ", qui donne au prélat tous les droits régaliens sur tout le Gévaudan épiscopal, dont celui de battre monnaie. Le monnayage épiscopal a du débuter vers 1161-1162, au moment de l'attribution des regalia ou un peu plus tard, vers 1170. L'évêque Aldebert tente de mettre à profit cette concession royale pour s'emparer des mines de son diocèse (1163-1170), mais il doit composer avec l'aristocratie locale, dont le prieuré d'Ispagnac. La dernière émission épiscopale pourrait se situer au début du $\mathrm{XIV}^{\mathrm{e}}$ siècle. Comme partout à la même époque, la reprise en main par le roi capétien réduit de façon irréversible les droits particuliers. En effet, la croisade contre les albigeois change la donne et introduit le roi de France. Une longue période de conflit oppose le sénéchal de Beaucaire, au service du roi, et l'évêque. Un acte de paréage, en 1307, entre les deux parties, partage le Gévaudan en trois domaines : le domaine

1. Les données historiques sont issues du mémoire de maîtrise de Sarah Laurent « Mines et métallurgie du plomb argentifère sur le Mont Lozère au Moyen Âge ( $\mathrm{XI}^{\mathrm{e}}-\mathrm{XV}^{\mathrm{e}}$ siècles) ", Lyon 2, 2005. 
de l'évêque, le domaine du roi et la terre commune, domaine des barons, administrée par des représentants épiscopaux et royaux au sein d'une cour commune. Ce découpage va rester en vigueur jusqu’à la Révolution Française.

En l'état des connaissances, il est certains que les sites à scories se répartissent sur les terres des barons du Tournel, de Florac et du Prieuré d'Ispagnac; il reste à mieux préciser qui possédaient les territoires où se trouvaient les mines. La question est primordiale pour comprendre la raison de l'arrêt de l'activité alors même que les gîtes métallifères ne sont pas épuisés.

Signalons que les barons de Florac étaient issus de la famille d'Anduze, originaire des Cévennes; cette famille a mené une véritable politique minière dans ses domaines, en particuliers dans le district minier d'Hierle, et a su résister aux vicomtes Trencavel sur ce front-là (Bailly-Maître, 2010, à paraître).

\section{LeS SITES 3 ET 8 ET LA MÉTALLURgIE DU PLOMB ARGENTIFÈRE}

Les sites à scories appartiennent à des typologies différentes : sites très apparents matérialisés par des épandages de scories sur lesquels la végétation est absente, sites enfouis uniquement signalés par quelques scories ou une différence de végétation.

Les premières campagnes de prospection ont été accompagnées de mini-sondages afin de tester le potentiel archéologique de plusieurs sites. La question étant de comprendre le fonctionnement des ateliers métallurgiques : typologie des fours, organisation spatiale des ateliers, impact environnemental, etc

Deux sites ont été choisis pour y ouvrir une fouille, tous deux sur le territoire de la commune de Saint-Etienne-duValdonnez (48). Il s'agit des sites 3 et 8 (fig. 1). L'apport respectif de ces deux ensembles est très inégal en fonction des thèmes abordés.

Le site 3 présentait plusieurs atouts. Tout d'abord, la structure visible en surface avait toutes les apparences d'un four et les masses importantes de scories laissaient espérer une activité métallurgique soutenue. Par ailleurs, l'équipe de botanistes et palynologues conduite par Jacques-Louis de Beaulieu était intéressée à travailler sur la tourbière qui se développe au contact du site $3^{2}$ (fig. 2).

Le site 8 se présentait sous la forme d'un épandage de scories sur $150 \mathrm{~m}$ de longueur fortement colonisé par des

2. Université Paul-Cézanne/CNRS - Aix-Marseille 3 - IMEP (Institut Méditerranéen d'Écologie et Paléoécologie). rumex (fig. 3). Comme le site 3, il était contigu à une tourbière, mais à l'inverse, aucun bâti n'était visible en surface. Au regard des résultats du site 3 dont les structures étaient très arasées, le site 8 offrait peut-être l'opportunité d'étudier des vestiges protégés par un recouvrement plus ou moins important.

\section{Les installations métallurgiques : les fours}

Seul le site 3 a livré des informations sur les fours. Les vestiges de deux, voire trois structures, étaient partiellement conservés sur une surface réduite (fig. 4).

Le four F1, (fig. 5), fouillé en 2001, est fait de blocs de grès et sa forme est grossièrement en $U$ renversé (environ $2 \mathrm{~m} 50$ de longueur pour $2 \mathrm{~m}$ de largeur extérieure). La cuve est en partie creusée dans la terre et en partie formée par une assise sommitale de blocs de grès. À l'intérieur de la cuve, un comblement de pierres recouvrait un remplissage de terre. Aucune trace de chauffe ou rubéfaction n'était conservée, ce qui posait la question de l'interprétation de cette structure. L'ensemble des indices contextuels - des scories noires et à patine blanche, des fragments de parois de four, ainsi que des couches charbonneuses - indiquait là un four métallurgique, mais un doute subsistait en raison de l'absence de preuve formelle dans la cuve même.

Une tranchée creusée immédiatement au nord du four F1 avait révélé une concentration de charbons de bois, un morceau de plomb coulé et enfin, une petite poche de cendres très compactées. L'étude des charbons a mis en évidence une singularité. Sur l'ensemble des sites alors échantillonnés, le bois charbonné pour les besoins de la métallurgie est le hêtre, ce qui est conforme à l'usage de la métallurgie ancienne. Dans le cas de la tranchée nord du site 3, l'assemblage contient $36 \%$ de hêtre pour $64 \%$ de pin (Allée et al., voir dans ce volume).

Le four FO se trouve à environ $3 \mathrm{~m}$ à l'ouest du premier, légèrement décalé vers le sud. La fouille a mis en évidence un amas de pierres reposant sur une couche très noire charbonneuse, d'une épaisseur de $14 \mathrm{~cm}$, riche en scories et fragments de parois de fours. L'hypothèse la plus vraisemblable est qu'il s'agit d'un four métallurgique F0, abandonné et démonté pour en récupérer les pierres. En l'absence de preuves incontestables, force est de se contenter d'hypothèses quant à l'interprétation de cette anomalie stratigraphique.

L'extension de la fouille en direction de la tourbière a montré que l'essentiel des vestiges se trouvait au sud du four F1. Sous l'humus et la terre ocre de colluvionnement, on rencontre une couche épaisse, noire, charbonneuse, très riche en scories noires et/ou à patine blanche et en parois de four (granite fondu). Cette couche recouvrait toute la 


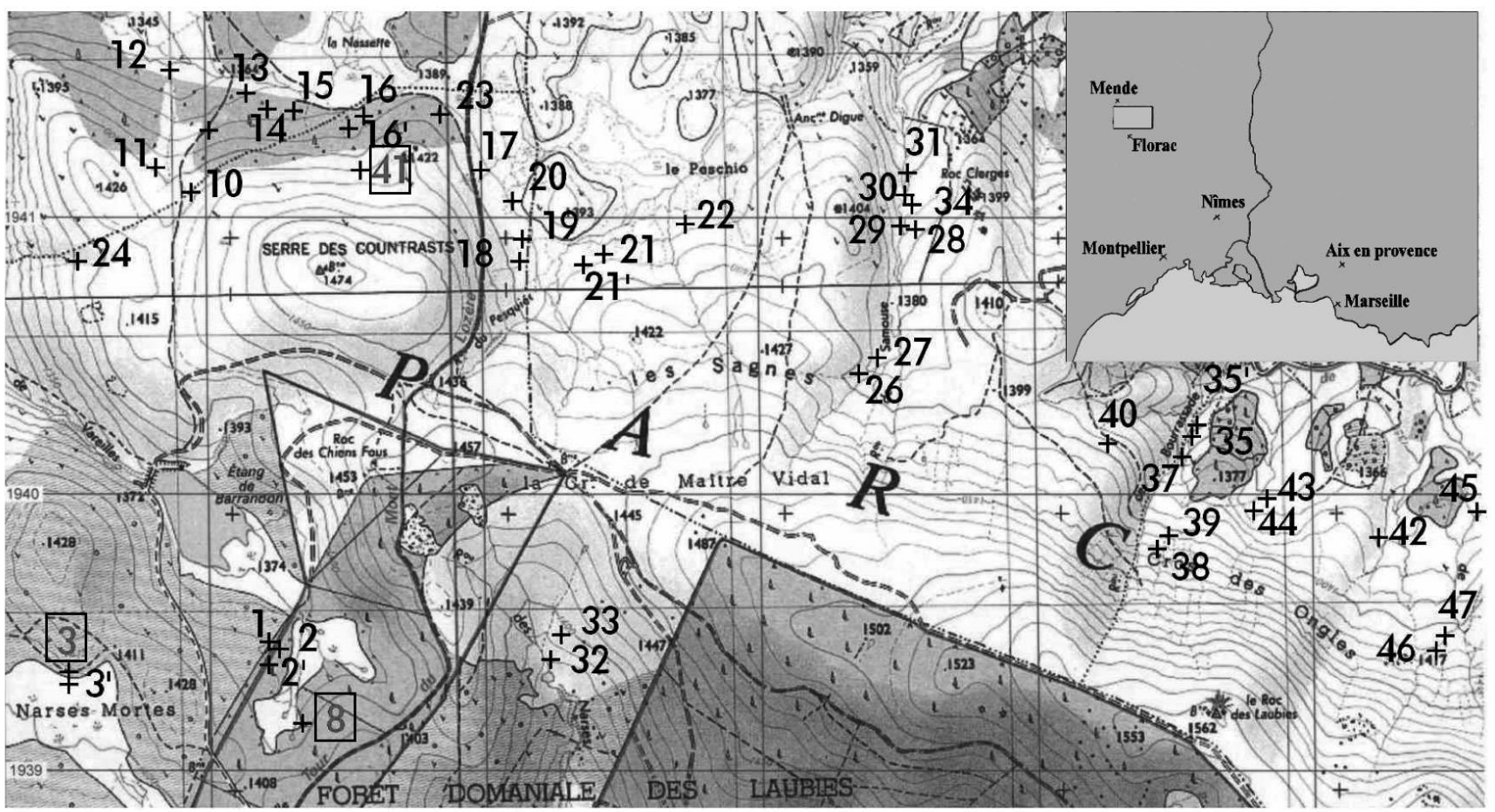

En encadré, sont indiqués les sites étudiés dans ce rapport Copyright IGN . Projection Lambert II étendu / NTF . Grille Lambert II étendu / NTF - FFRP pour les itinéraires et sentiers de randonnees GR®, GRPQ, PR@

Figure 1: Localisation des sites 3 et 8.

Figure 1: Location of sites 3 and 8.

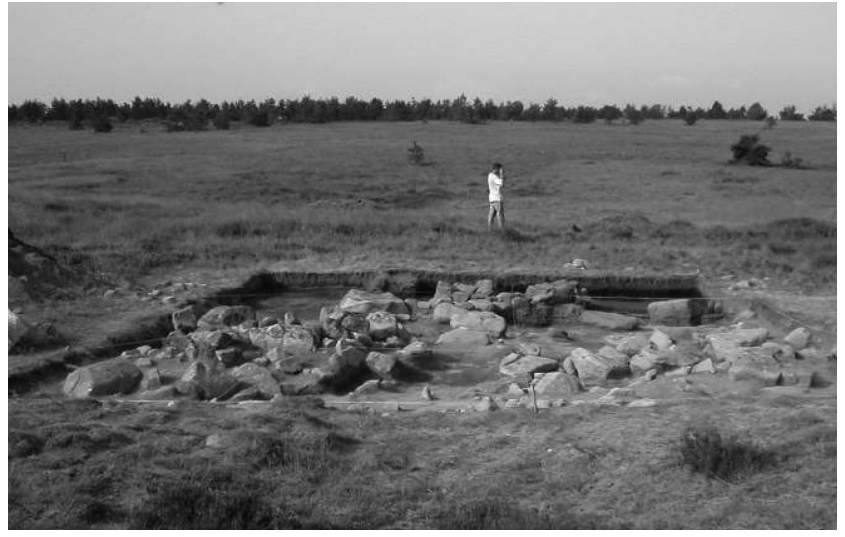

Figure 2 : Site 3 : vue d'ensemble en bordure des Narses Mortes. Figure 2: Site 3: overview on the edge of dead Narses.

surface dégagée et entourait le four F2 dont quelques pierres affleuraient.

Le four F2 est constitué d'un double cordon de blocs de grès plus ou moins épais (fig. 4, 6). L'ensemble prend la forme d'un $U$ renversé, orienté approximativement nordsud. Les dimensions extérieures sont de $3 \mathrm{~m}$ de longueur

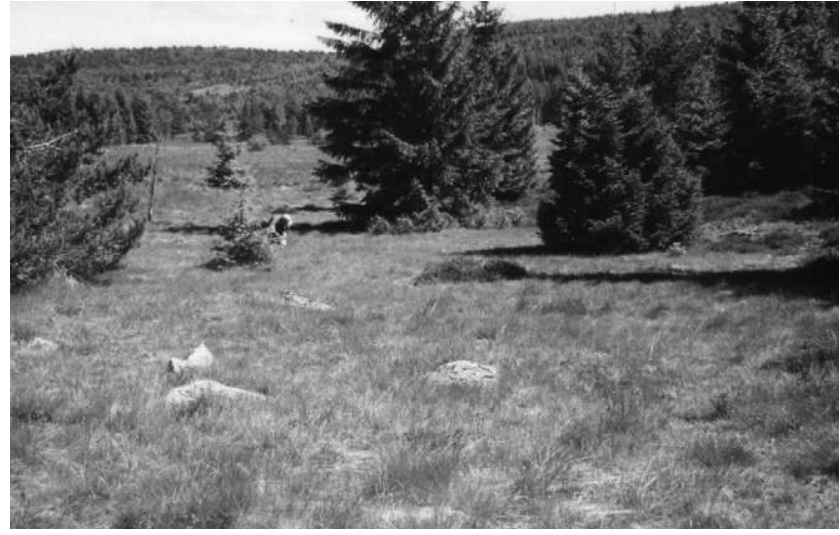

Figure 3 : Site 8 : vue générale, les rumex donnent au site sa couleur rouge.

Figure 3: Site 8: overview, the rumex give a red color.

pour $2 \mathrm{~m}$ de largeur et les mesures intérieures de la cuve sont de $1 \mathrm{~m} 50$ de longueur pour $0 \mathrm{~m} 80$ de largeur. F2 est en tous points identique à $\mathrm{F} 1$ : même mode de construction, même module, même absence de rubéfaction, même quasiabsence de scories à l'intérieur de la cuve. Cette découverte conforte l'identification de F1 comme un four métallur- 


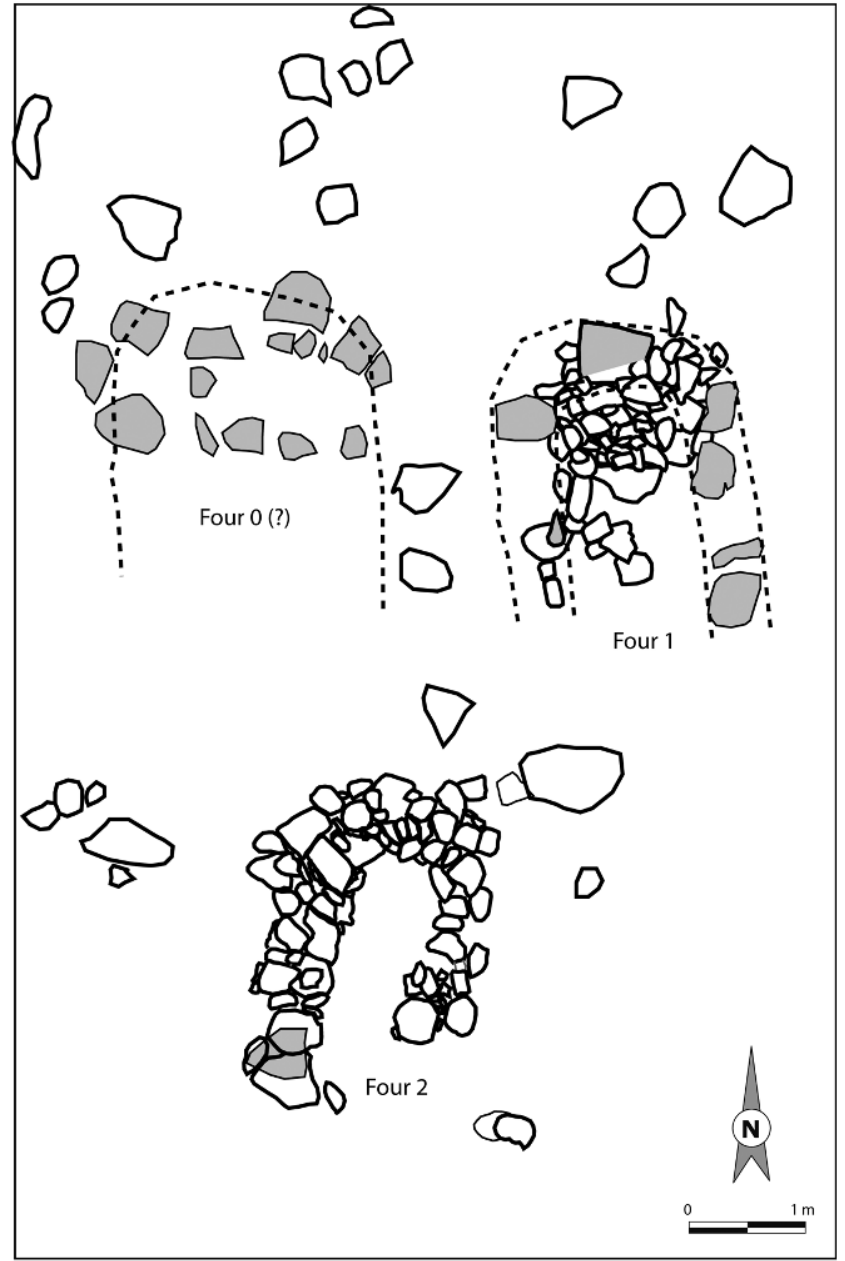

Figure 4 : Site 3 : plan général des structures. Figure 4: Site 3: général plan structures.

gique. Une très importante poche de scories noires et/ou à patine blanche, et de parois de four était concentrée contre le mur externe de l'abside du four, sans terre interstitielle. Il s'agit certainement d'une aire de rejet résultant du nettoyage de la structure au cours de son fonctionnement.

La fouille de l'intérieur de la cuve a montré plusieurs niveaux. Après deux horizons sans liens avec le fonctionnement du four, une couche noire est apparue, de plus en plus riche en scories, dont certaines d'un module supérieur à $5 \mathrm{~cm}$. Cette couche, dont l'épaisseur varie, est présente à l'intérieur et tout autour du four F2. Son interprétation chronologique est difficile car la structure semble installé sur et dans cette couche. Cependant, ce niveau correspond, sans aucun doute, au fonctionnement d'un four métallurgique. Il ne semble pas qu'un aménagement antérieur a F2 ait été installé au même endroit; une tranchée creusée à l'ouest de la structure montre que la couche noire tend à

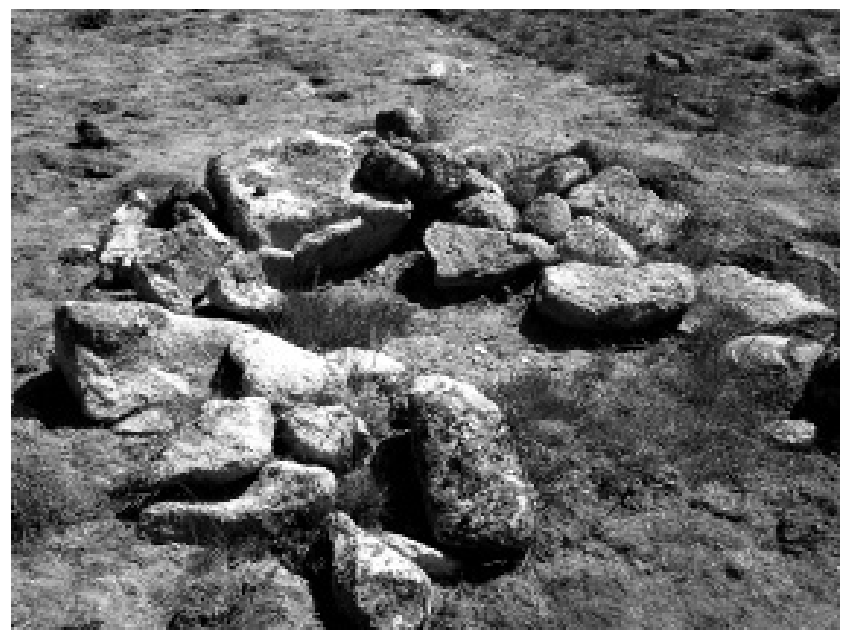

Figure 5 : Site 3 : four F1 avant la fouille.

Figure 5: Site 3: furnace F1 before the excavations.

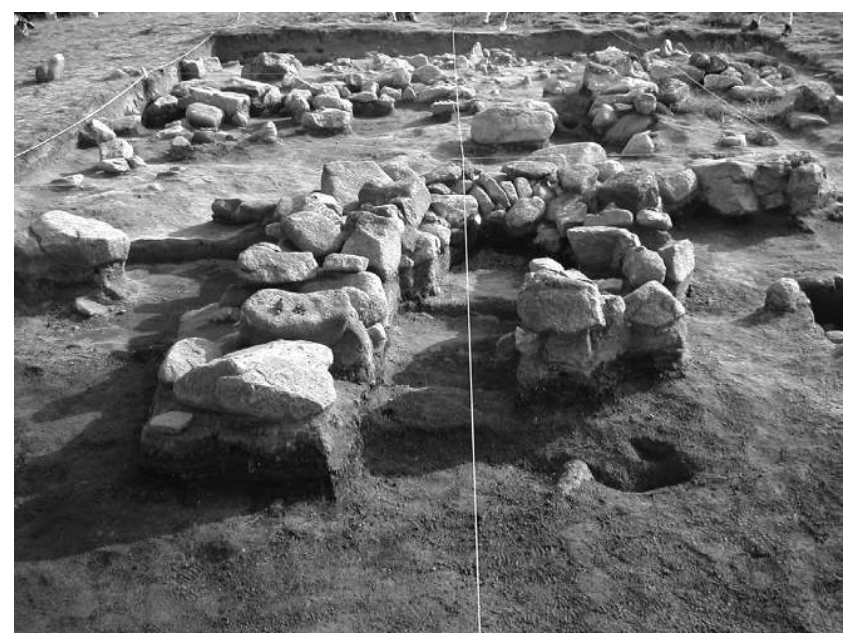

Figure 6: (Voir planche couleur) Site 3 : four F2.

Figure 6: (See colour plate) Site 3: furnace F2.

s'épaissir vers son contact avec F2 et qu'elle s'amenuise du sud vers le nord, ce qui confirme qu'elle correspond bien au fonctionnement du four F2 et n'est pas liée au four F0 ni à $\mathrm{F} 1$.

Larchitecture de ce four est très rudimentaire. La partie supérieure est formée de deux couronnes de pierres. La couronne extérieure, incomplète, ne comprend qu'une seule assise, et repose sur la couche noire. La couronne intérieure, qui forme la cuve, est construite avec davantage de soin, les pierres sont bien ajustées; elle comprend parfois deux assises de blocs. Il semble que les blocs extérieurs servent simplement de contrefort et d'isolant thermique. En arrière de l'abside extérieure se dessinait un court petit chenal rempli d'une argile brune. Il s'agit peut-être de l'emplacement 
de la tuyère, comblé après abandon de la structure, mais rien n'était visible à ce niveau à l'intérieur du four.

La poursuite du décapage en avant du four a mis en évidence la stratigraphie jusqu'au sol d'installation de la structure métallurgique. Sous un millefeuille de terre argileuse grise, noire et brune, en strates très fines, contenant également quelques nodules de couleurs rouille, mais aucune scorie, d'une épaisseur de 3 à $4 \mathrm{~cm}$, se développe une fine strate noire, très irrégulière, creusée de petites dépressions, passant également sous la structure F2. Enfin, on atteint une couche très organique, qui est en fait la tourbière sur laquelle les métallurgistes se sont installés (fig. 7). Ce niveau a été échantillonné dans son épaisseur pour les études paléo-environnementales, car il s'agit du niveau de tourbe en place au moment de la première installation métallurgique.

En conclusion sur la stratigraphie, on remarque que le fonctionnement des fours a engendré des niveaux charbonneux épais, riches en déchets de métallurgie. Ces niveaux peuvent être entrecoupés de fines strates grises, résultat possible de recharges de sol de circulation ou bien de terre rapportée sous les semelles des métallurgistes.

Le caractère très arasé des constructions mises au jour sur le site 3 incite à penser que seules les bases des fours étaient conservées, ce qui ne permet pas d'établir une typologie de ces structures métallurgiques.

\section{L'organisation spatiale des ateliers : à la recherche des aménagements connexes}

L'emploi du mot atelier se comprend, ici, comme un ensemble de structures liées au traitement du minerai. Dans

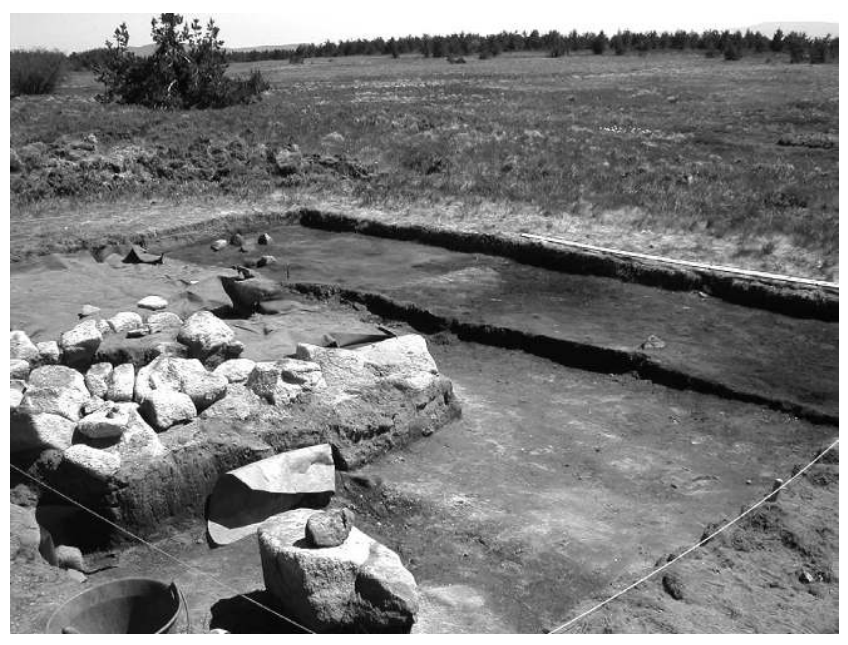

Figure 7 : Site 3 : position relative du four F2 et de la tourbière. Figure 7: Site 3: position between the furnace four F2 and the peat bog. aucun des sites inventoriés il ne semble qu'il y ait de bâtiment; tout se passe en aire ouverte.

Un atelier de métallurgie peut comporter divers d'aménagements utiles à son fonctionnement : préparation mécanique ou tri du minerai, stockage du minerai et/ou du combustible, appentis pour les ouvriers...

Ces aménagements ont été recherchés aux abords des fours du site 3 dans les quatre directions. L'extension ouest à immédiatement mis en évidence le substrat granitique. Lextension nord, en arrière du four $\mathrm{F} 1$ a livré, après un espace vide de vestiges, des indices - charbon de bois et coulure " plombeuse "- appartenant très certainement à un four situé en amont de F1. L'extension orientale correspondait à une aire de rejet de scories résultant d'un nettoyage du four F1; le sol avait été induré par le déversement de matières chaudes. Les ouvriers rejetaient leurs déchets au sortir du four, sans prendre la peine d'entretenir des espaces de passage et de manutention.

La poursuite de la fouille au sud du four F2 a montré que les métallurgistes se sont implantés en bordure de la tourbière, à un moment où les eaux étaient basses, sans aménagement de sol et sans travaux d'assainissement préalables.

La fouille du site 8 n'a pas permis de retrouver de four, mais deux éléments liés aux opérations qui se pratiquaient aux abords des fours.

Le premier concerne un possible tri du minerai. Un excès de $\mathrm{Cu}$ a été détecté dans le sol d'atelier $8^{3}$. Il peut s'expliquer par un dernier tri du minerai sur place, afin d'éliminer les chalcopyrites considérées comme un "poison " car elles favorisent l'apparition de mattes.

Le second a trait au combustible. À la fin de la campagne 2006, en limite nord-ouest de l'aire ouverte, une fosse apparaissait, scellée par un important amas de scories. L'extension de la fouille en 2007 n'a pas permis d'atteindre les limites de cette fosse vers le nord. Au terme de la campagne, elle occupait une surface de près de $3 \mathrm{~m}^{2}$ sur son rebord supérieur. Son remplissage atteignait un maximum de $50 \mathrm{~cm}$ de profondeur; il était constitué quasi exclusivement de charbon de bois, à l'exclusion de quelques rares scories (moins d'une dizaine). Un prélèvement massif des sédiments a été réalisé pour une étude anthracologique et datation (en cours). D'ores et déjà, un litage très fin apparaissait, formant un millefeuille qu'il n'était pas possible de relever, mais qui sera déterminé en laboratoire. Ce litage témoigne d'un remplissage par apports successifs qui semblent provenir d'une source située en amont, au sud-est, c'est-à-dire dans le bois qui jouxte le site. Pour l'heure, cette fosse à charbon de bois est interprétée comme une

3. Étude réalisée par Sandrine Baron. 
réserve de combustible, en attente de précisions archéométriques.

L'aire ouverte sur le site 8 était recouverte par une strate formée d'une fine cendre grise qui a été interprétée, dans un premier temps, comme le produit de défournage des structures métallurgiques. Aujourd'hui, l'identification est moins certaine en raison de son extension. Des prélèvements ont été réalisés pour analyses.

\section{Que sait-on sur l'organisation spatiale des ateliers?}

Seule la fouille extensive du site 3 permet quelques remarques :

- trois fours ont été retrouvés, assez proches les uns des autres. Pour autant, ils n'ont pas fonctionné en batterie puisqu'il s'établit une chronologie relative assez nette entre les différentes structures;

- ces fours se sont installés sur le rivage de la tourbière des Narses Mortes, au cours d'une période de sècheresse, comme l'a montré la stratigraphie. Les niveaux résultant de l'activité industrielle reposent directement sur une couche de tourbe;

- l'extension de l'aire de fouille dans toutes les directions autour des structures métallurgiques n'a pas permis de retrouver d'aménagements annexes, liés au fonctionnement des fours, comme des aires de stockage de minerai ou de combustible. On peut imaginer que le minerai était préparé en dehors du site métallurgique, les emplacements n'ont pas été localisés.

Ces observations ne peuvent pas être généralisées, en raison du trop faible nombre de sites fouillés. D'autre part, le site 8 livre des informations différentes sur l'organisation spatiale des aires de travail; ces informations sont cependant trop fugaces pour le moment pour en tirer des conclusions.

\section{Des ateliers pour quelle métallurgie, avec quel minerai et à quelle époque?}

Plusieurs approches archéométriques ont pris comme objet les sites 3 et 8 . Les résultats de ces travaux ont déjà été publiés par ailleurs (bibliographie générale, voir ce volume); les lignes qui suivent ne sont qu'un bref rappel des principaux apports de ces recherches, de façon à présenter l'ensemble des données fournies par ces deux sites.

\section{Les analyses des scories}

Les deux sites ont livré des scories noires vitreuses, des scories à patine blanche, des scories grenat et enfin, des parois de fours représentées par des granites très vitrifiés (fig. 8). Les scories à patine blanche étaient très friables et avaient une forte tendance à se briser au moment de la fouille. Une analyse géochimique et pétrologique a été réalisée sur ces résidus. Les résultats confirment que les scories étudiées résultent bien d'une métallurgie du plomb, probablement argentifère. Le procédé métallurgique mis en œuvre est dit par " grillage et réduction ", procédé le plus couramment employé avant les $\mathrm{XIV}^{\mathrm{e}}$-XV $\mathrm{X}^{\mathrm{e}}$ siècles (Mahé-le Carlier, Ploquin, Fluck, voir ce volume).

\section{Les isotopes du plomb, origine des minerais et pollution}

Lapproche isotopique a porté sur plusieurs types de vestiges (Baron, Ploquin, voir ce volume), avec plusieurs objectifs :

Point 1 : rechercher l'origine possible des minerais,

Point 2 : déterminer si le minerai provenait de plusieurs mines aux caractéristiques très différentes,

Point 3 : quantifier la pollution engendrée par l'activité métallurgique.

Pour les points 1 et 2 , les sites 8 et 3 ont concentré le plus d'analyses isotopiques; les isotopes ont été recherchés sur des billes de plomb contenues dans les scories. Sur le site 3, les rapports forment un seul noyau; sur le site 8, deux noyaux distincts apparaissent. Est-ce à dire qu'il y a eu deux sources d'approvisionnement? Dans ce cas, elles sont toutes deux situées dans le potentiel de mines qui se trouvent dans le secteur sud-ouest : seule la grande précision atteinte par l'analyse permet de faire le distinguo. L'origine vraisemblable des minerais serait le secteur Montmirat - Nozières - Les Bondons.

Pour le point 3, le plomb et les isotopes du plomb ont également été dosés sur plusieurs spécimens de niveaux. Le site 8 a servi de terrain expérimental pour plusieurs approches archéométriques : des analyses isotopiques à partir des scories; des mesures de pollution des sols d'atelier; des mesures de pollution sur la végétation. Concernant les sols d'atelier, ces derniers présentent des teneurs en métaux lourds - $\mathrm{Pb}, \mathrm{As}, \mathrm{Cu}$ - très élevées comparativement au bruit de fond local des granites, du même ordre de grandeur que des sites industriels... mais sur une surface moindre (Baron, Ploquin, voir dans ce volume $^{4}$. Concernant l'impact sur la végétation : «Afin de tester la part du plomb anthropique dans la végétation actuelle proche d'un atelier médiéval (site 8), trois bouleaux ont été échantillonnés... bouleau 1 sur l'épandage du site, bouleau 2 juste en aval du site et le bouleau 3 à $100 \mathrm{~m}$ à l'écart de la circulation des eaux et sédiments. » " Les compositions isotopiques des bois du bouleau 3 (B-3), situé hors de l'épandage de pollution, contiennent encore 70 à $80 \%$ de son plomb hérité de la métallurgie médiévale et les écorces en contiennent 5 à $15 \%$ » (Baron, Ploquin, voir ce volume).

4. S. Baron Traçabilité et évolution d'une pollution métallurgique médiévale de plomb argentifere sur le Mont-Lozère, Univ. Montpellier (Maison de l'Eau), travaux au CRPG, direction : $\mathrm{M}^{\mathrm{me}} \mathrm{F}$. Elbaz-Poulichet (Montpellier), thèse soutenue en 2005. 


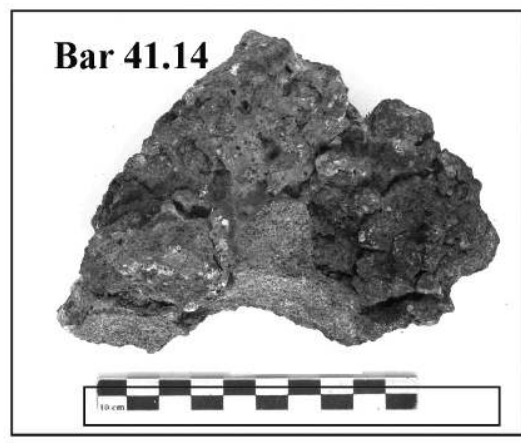

Paroi de four

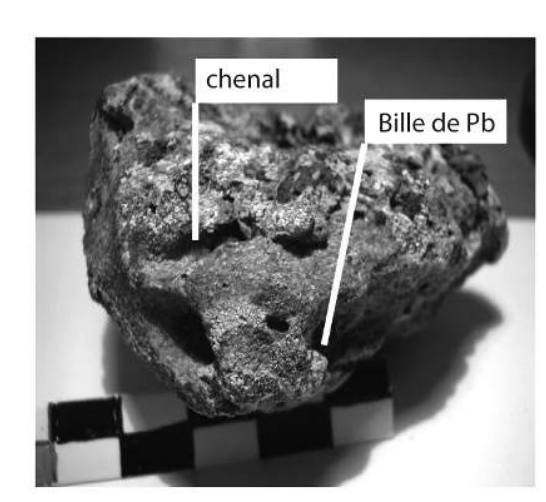

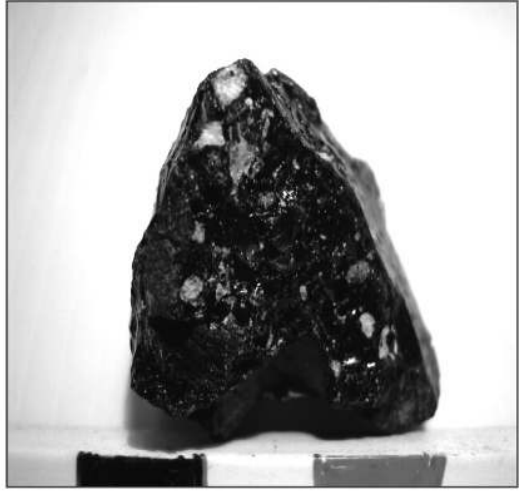

Scorie noire

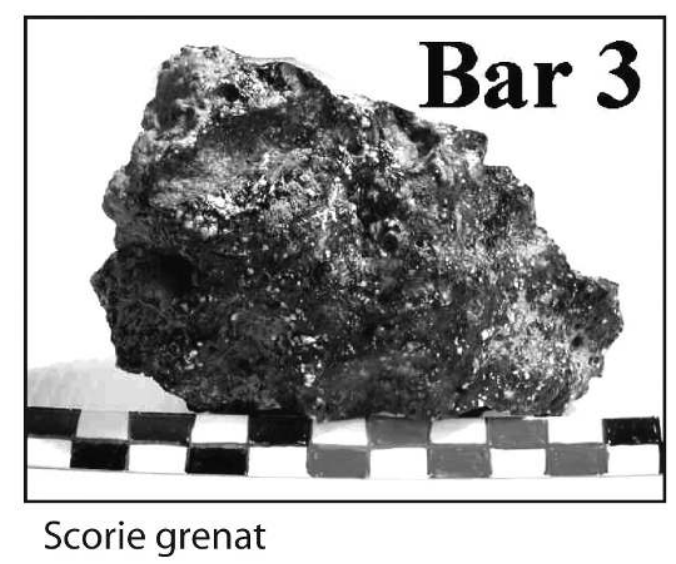

Scorie grenat

Figure 8 : (Voir planche couleur) Échantillonnage des différents types de rejets de la métallurgie.

Figure 8: (See colour plate) A selection of different types of releases of metallurgy.

\section{Chronologie}

Trois datations ont été réalisées sur le site 3 et ses abords immédiats ${ }^{5}$ :

- La première porte sur des charbons de bois contenus dans l'US4 du site archéologique, strate correspondant au fonctionnement des fours. La fourchette de plus forte probabilité est $840+/-30$, soit en âge calibré $1155-1265 \mathrm{cal}$ $\mathrm{AD}$, mais cette seule date reste à confirmer par la datation d'autres échantillons ${ }^{6}$.

- La seconde a été faite sur une branche de chêne trouvée à la base de la tourbière, au sud du site. La datation obtenue est +/- 2525 BP, soit en âge calibré 695-539 avant notre ère ${ }^{7}$.

- La troisième porte sur le charbon de bois prélevé dans le sondage pratiqué en amont, au nord du four F1. Le charbon de bois y formait une poche nette, en limite orientale de la tranchée. Il n'était pas associé à des scories, mais à un morceau de plomb coulé et à une poche de cendres très compactées. L'étude anthracologique des charbons de bois a fait

5. Poznan Radiocarbon Laboratory (Poznam - Pologne).

6. Poznam, ref. POZ 7047.

7. Poznam, ref. POZ 7042. apparaître une anomalie puisque l'assemblage contient $36 \%$ de hêtre pour $64 \%$ de pin, contrairement aux autres sites où le hêtre est seul représenté. L'âge obtenu sur ces charbons de bois confirme la singularité de cet ensemble puisqu'il est comparable à la branche de chêne.

Ces deux dernières datations posent le problème d'une métallurgie antérieure au Moyen Âge.

Concernant les structures mises au jour sur le site 3 , qui appartiennent toutes trois à la période médiévale, une chronologie relative peut s'établir : fonctionnement du four F0, matérialisé par un amas de pierres et un environnement riche en charbons de bois et déchets métallurgiques; puis abandon et démontage du four F0 et construction du four F1 dont les structures bâties sont bien conservées, mais qui a laissé peu de traces de son fonctionnement; enfin, construction du four F2, le dernier à fonctionner sur la zone étudiée, qui a laissé des couches importantes. On ne peut donc pas parler de fonctionnement de fors en batterie, ils emblent se succéder dans le temps.

Seules deux datations ont été réalisées pour le site 8. La première porte sur des charbons de bois, mêlés aux scories, 
prélevés dans des dépôts colluviaux (US2) qui recouvrent immédiatement les structures; la datation obtenue est 930 +/- 30, soit en âge calibré $1025-1168 \mathrm{cal} \mathrm{AD}^{8}$. La seconde porte également sur des charbons de bois mêlés à des scories, cette fois prélevés à la base du site (US9); la datation obtenue est $930+/$ - 30, soit en âge calibré 1025-1168 cal AD 9 .

\section{Métallurgie et PalÉO-ENVIRONNEMENT}

Plusieurs approches transdisciplinaires permettent de mesurer l'impact paléo-environnemental de l'activité métallurgique ancienne. Les principaux apports concernent l'impact sur la forêt (combustible) (Allée et al., voir ce volume), la pollution (Baron et al., voir ce volume) et le contexte paléo-climatique au moment de l'installation des métallurgistes au Moyen Âge.

\section{Le combustible}

Les charbons de bois recueillis sur les sites 3 et 8 résultent du charbonnage du hêtre, comme sur la quasi-totalité des sites de métallurgie et de charbonnières (Allée $e t$ al., voir dans ce volume). Seule discordance dans ce tableau, les échantillons provenant de la tranchée creusée dans l'axe et au nord du four F1 du site 3. À $3 \mathrm{~m}$ de l'abside du four, une poche de charbon de bois, assez concentrée a été entièrement prélevée pour datation et détermination anthracologique. L'étude des charbons a révélé un assemblage contenant $64 \%$ de pin. Cette anomalie est certainement à mettre en relation avec la datation haute obtenue sur ces mêmes charbons de bois (voir ci-dessus).

\section{Le paléo-climat}

C'est encore une fois la tourbière du site 3 - les Narses Mortes - qui livre l'essentiel des informations. L'ouverture de l'aire de fouille en avant du four F2 a clairement mis en évidence que la couche de tourbe vient mourir sous les bases du four pour s'épaissir en direction du sud. Elle repose directement sur l'arène granitique. Un sondage pratiqué, en 2002, dans la tourbière en aval d'un fossé de drainage, a montré qu'à quelques centimètres au-dessus du saprolithe (fond de la tourbière, arénacé ici, altéré par la tourbière), plusieurs morceaux de branches étaient disposés à plat : bouleau, genévrier, et, plus spectaculaire, six morceaux apparemment " épointés » de sapin. Le caractère anthropique de

8. Ref. 2373

9. Pozman, ref. Poz 7043 . l'épointage n'a pas été établi lors de l'examen fait à l'INRANancy. L'examen en lame mince a conduit à la conclusion "sapin ayant poussé en condition d'altitude, marques de gel ». En juin 2003, un nouveau sondage fait à la demande et par les palynologues, est tout à fait analogue; une branche de chêne, de la taille du bras, gisait au même niveau profond : son radiocarbone a donné $2510 \mathrm{BP}^{10}$. D'après les palynologues, le site 3 est à $300 \mathrm{~m}$ au-dessus de la limite altimétrique du chêne : ces branches auraient donc été amenées par l'homme à La Tène.

Une dernière tranchée a été pratiquée, en 2005, au sud du four F2, dans la tourbière de part et d'autre du fossé de drainage. Quelques scories ont été trouvées dans les racines superficielles. À quelques centimètres sous le sommet de la tourbe, des fragments pluri-centimétriques étaient disposés à plat. Ils sont plus abondants au sud du fossé. Sous cette surface à " écorces" (à $10 \mathrm{~cm}$ sous le sommet de la tourbe) est apparue une structure à tendance polygonale de type mottes et fentes de dessiccation. Une fois reconnue, elle a pu être suivie de façon discontinue, de part et d'autre du fossé. C'est dans les fentes que les quelques scories trouvées sont localisées, ainsi que des charbons de bois. Malgré la difficulté à suivre cette surface, il semble bien qu'elle corresponde, dans la fouille, à la surface gondolée et indurée, gris brun à noire du fonctionnement de F2 : ce qui confirme que l'atelier s'est installé en bordure de tourbière lors d'une période sèche.

\section{Conclusion}

Le site 3 des Narses Mortes et le site 8 laissent encore beaucoup de questions en suspens quant au processus de transformation métallurgique du plomb argentifère et aux structures mises en place dans cet objectif. Mais ce sont les premiers du genre à être fouillés et les informations recueillies sont donc essentielles, même si un long chemin reste à parcourir pour bien comprendre toute la chaîne opératoire. La fouille de structures comparables s'avère d'ores et déjà nécessaire et le secteur du Mont Lozère, sur lequel s'appuie le PCR, en compte un certain nombre à fort potentiel archéologique.

Au niveau de l'organisation du travail, ces fours, faciles à construire, fonctionnaient très certainement peu de temps, étaient démontés et reconstruits à proximité les uns des autres. On peut restituer un système saisonnier, avec des fours qui tournent dans cette bande altitudinale qui leur permet de compter sur des réserves de combustible. La mise en évidence, sur le site 3, de plusieurs structures qui se sont

10. Poznan Radiocarbon Laboratory (Poznam - Pologne). 
succédées dans le temps, infirme l'hypothèse de fours en batterie au bénéfice de fours rudimentaires fonctionnant de façon plus ou moins isolée, mais cependant au sein d'un vaste complexe métallurgique.

La démarche coordonnée historique, archéologique, archéométrique et paléo-environnementale entretient un dialogue constant entre ce qui a permis le développement de cette activité (minerais, combustible, demande économique), le fonctionnement de l'activité et enfin, son impact sur le territoire. Ainsi, la carotte prélevée dans la tourbière des Narses Mortes a mis en évidence un impact de l'activité métallurgique beaucoup plus important pour la période gauloise que pour le Moyen Âge, seul visible actuellement sur le Mont Lozère. Ce pourrait être la première confirmation archéométrique de l'exploitation de l'argent par les Gabales, une nouvelle piste de recherche...

\section{Bibliographie}

Agricola, G., 1987. De re metallica, Bâle, Forben et Bischoff, 1557, réed. trad. France-Lanord, A., Thionville, Klopp, 508 p.

Bailly-Maître, M.-Ch. et Gauché S., 2002. Une approche du droit minier en Dauphiné (XIv ${ }^{\mathrm{e}}$-début $\mathrm{Xv}^{\mathrm{e}}$ siècle), La Pierre et l'Écrit, n 13 , p. 97-114.

Bailly-Maître, M.-Ch., 2002. L'Argent. Du minerai au pouvoir dans la France médiévale, Paris, Picard, coll. «Espaces médiévaux ", $264 \mathrm{p}$.

Bailly-Maître, M.-Ch. et Dhénin, M., 2004. Ateliers monétaires et mines d'argent dans les Alpes ( $\mathrm{x}^{\mathrm{e}}-\mathrm{Xv}^{\mathrm{e}}$ siècle). Histoire, archéologie, numismatique et archéométrie, Archéologie Médiévale, tome 34, Paris, CNRS éditions, p. 43-64.

Bailly-Maître, M.-Ch., 2004. Les agglomérations minières au Moyen Âge en Europe occidentale, Naissance et développement des villes minières en Europe, colloque international, Liévin-Lens, 2002, Arras/Paris, Artois Presses Université/PUP Sorbonne, collection " Histoire », p. 215-226.

Bailly-Maître, M.-Ch. et Poisson, J.-M., 2007. Mines et pouvoir au Moyen Âge, actes de la Table Ronde Mines et pouvoir châtelain, Lyon, mai 2002, coll. d'Histoire et d'Archéologie médiévales, Lyon, PUL, 213 p.

Bailly-Maître, M.-Ch., 2006. Plomb, cuivre, argent et zinc. Histoire du district minier des Malines (St Laurent-le-Minier - Gard) du XII ${ }^{\mathrm{e}}$ au Xxe siècle, Savoirs et savoir-faire industriels en Méditerranée occidentale (XVI- $X X^{\mathrm{e}}$ siècle), colloque international Méditerranéen, Corte, juin 2000, co-éd. Museu di Corsica, Corte et Journal de la Corse, Ajaccio, p. 89-103.

Bailly-Maître, M.-Ch., 2008. Les paysages miniers médiévaux en France : essai de restitution à partir des données de l'archéologie et de l'archéométrie, Proceeding on Le Mans COST A27 conférence, Marqueurs des paysages et systèmes socio-économiques de la construction des paysages pré-industriels à leur perception par les sociétés contemporaines, Le Mans, décembre 2006, Presses universitaire de Rennes, Documents Archéologiques 1, mars, p. 89-98.

Bailly-Maître, M.-Ch. et Dillman, Ph., sous presse. Mines et métallurgies au Moyen Âge.Une thématique de recherche récente, actes du colloque Trente d'archéologie médiévale en France : un bilan pour un avenir, Vincennes, juin 2006, Publications du Centre de recherches archéologiques et historiques de Caen.

Bailly-Maître, M.-Ch., 2009. Les richesses minières au service du pouvoir. Politique comparée des Anduze et des Trencavel dans l'arrière-pays cévenol, actes du colloque Autour du sac de Béziers : Richesses et malheurs du Languedoc au XIII siècle, URBI, juin.

BARON S., 2005. Traçabilité et évolution d'une pollution métallurgique médiévale de plomb argentifere sur le Mont-Lozère, doctorat d'Université Montpellier II, discipline Géochimie de l'Environnement.

Baron, S., Lavoie, M., Ploquin, A., Carignan, J., Pulido, M. et Beaulieu, J. L. de, 2005. Record of Metal Workshops in Peat Deposits: History and Environmental Impact on the MontLozère Massif (France), Environmental Science and Technology, 39, p. 5131-5140.

Biringuccio, V., 1540, 1990. De la Pirotechnia. The Classic Sixteenth-Century Treatise on Metals and Metallurgy, ed. C. Stanley and M.T. Gnudi, New York, Dover Publications, p. 477.

Braunstein, Ph., 1990. Le travail minier au Moyen-Âge d'après les sources réglementaires, colloque Le travail au Moyen Âge, une approche interdisciplinaire, publication de l'Institut d'Études Médiévales, Université Catholique de Louvain, Louvain-la-Neuve.

Caliste, L., 2005. Ramondens, une forêt de la fin du Moyen Âge. De la formation d'un patrimoine à son exploitation industrielle (XIIt $-X V^{e}$ siècles), université Paris 8-Saint Denis, mémoire de maîtrise sous la direction de C. Verna, $251 \mathrm{p}$.

Fluzin, Ph., Ploquin, A. et Serneels, V., 2000. Archéométrie des déchets de production sidérurgique, Moyens et méthodes d'identification des différents éléments de la chaîne opératoire directe, Gallia, 57, p. 101-121.

Francovich, R. 1985. Per la storia della metallurgia e dell'insediamento medievale sulla costa toscana: lo scavo del villaggio minerario di San Silvestro, Rassegna di Archeologia, IV, p. 359-382.

Francovich, R., 1985. Un villaggio di minatori e fonditori di metalli nella Toscana del medioevo: San Silvestro (Campiglia Marittima), Archeologia Medievale, XII, p. 313-402.

Hesse, P.-J., 1968. La mine et les mineurs en France de 1300 à 1500 , thèse de droit, Paris, 3 volumes. 
ISAMBERT, F., 1821-1833. Recueil général des anciennes lois françaises, 29 vol., Paris, t. VII.

LAURENT, S., 2005. Mines et métallurgie du plomb argentifère sur le Mont Lozère au Moyen Âge ( $\mathrm{XI}^{\mathrm{e}}-\mathrm{XV}^{\mathrm{e}}$ siècles), Lyon 2.

Lavoie, M., Pulido, M., Baron, S., Ploquin, A., Beaulieu, J.-L., de, Bailly-Maître, M.-C., Carignan, J., Allée, P., Laurent, S., Mahé-Le Carlier, C. et Peytavin, J., 2005. Le plomb argentifère ancien du Mont Lozère (Massif Central) : une approche historique, géologique et paléoenvironnementale. Gestion des paléoenvironnements et stratégies d'exploitation des milieux de moyenne montagne, Actes du séminaire inter- national de Pierrefort, juin 2003 (F. Sourmely et Y. Miras, éditeurs), Annales Littéraires, Presses Universitaires FrancComtoises, Série "Environnement, sociétés et archéologie ", Besançon, p. 127-135.

Prassl, J.-A., 1997. " Exploitation antique dans le secteur minier du Bleymard (Lozère) ", Mines et métallurgies de la préhistoire au Moyen Âge en Languedoc-Roussillon et régions périphériques, Archéologie en Languedoc, Revue de la Fédération Archéologique de l'Hérault, 21, p. 217-224.

SchwaZERBERGBuch, 1556, 1988. coprigth by Verlag Gluckaüf GMBH, Essen. 